PROCEEDINGS OF THE

AMERICAN MATHEMATICAL SOCIETY

Volume 132, Number 1, Pages 1-6

S 0002-9939(03)07240-X

Article electronically published on August 13, 2003

\title{
SOLUTION TO A PROBLEM OF S. PAYNE
}

\author{
XIANG-DONG HOU
}

(Communicated by David E. Rohrlich)

\begin{abstract}
A problem posed by S. Payne calls for determination of all linearized polynomials $f(x) \in \mathbb{F}_{2^{n}}[x]$ such that $f(x)$ and $f(x) / x$ are permutations of $\mathbb{F}_{2^{n}}$ and $\mathbb{F}_{2^{n}}^{*}$ respectively. We show that such polynomials are exactly of the form $f(x)=a x^{2^{k}}$ with $a \in \mathbb{F}_{2^{n}}^{*}$ and $(k, n)=1$. In fact, we solve a $q$-ary version of Payne's problem.
\end{abstract}

\section{INTRODUCTION}

Let $\mathbb{F}_{2^{n}}$ be the finite field with $2^{n}$ elements. In 1971, S. Payne posed the following problem [6]:

Problem 1.1. Determine all linearized polynomials

$$
f(x)=a_{0} x+a_{1} x^{2}+\cdots+a_{n-1} x^{2^{n-1}} \in \mathbb{F}_{2^{n}}[x]
$$

such that $f(x)$ is a permutation polynomial of $\mathbb{F}_{2^{n}}$ and

$$
\frac{f(x)}{x}=a_{0}+a_{1} x^{2-1}+\cdots+a_{n-1} x^{2^{n-1}-1}
$$

is a permutation of $\mathbb{F}_{2^{n}}^{*}$.

Problem 1.1 originated from projective geometry. In fact, the polynomials in Problem 1.1 give rise to ovoids in the projective plane $\mathrm{PG}\left(2,2^{n}\right)$. (Cf. [2], p. 50 and [5].) Obviously, if $a \in \mathbb{F}_{2^{n}}^{*}$ and $k$ is a positive integer such that $(k, n)=1$, then $f(x)=a x^{2^{k}}$ satisfies the requirements in Problem 1.1. However, as noted in [6], no other linearized polynomials with the same properties are known. In this paper, we will show that $f(x)=a x^{2^{k}}\left(a \in \mathbb{F}_{2^{n}}^{*},(k, n)=1\right)$ are the only polynomials in Problem 1.1 In general, for any $\mathbb{F}_{q^{-}}$-linear map $f: \mathbb{F}_{q^{n}} \rightarrow \mathbb{F}_{q^{n}}$, we say that $f(x) / x$ is a permutation of $\mathbb{F}_{q^{n}}^{*} / \mathbb{F}_{q}^{*}$ if given any $\alpha \in \mathbb{F}_{q^{n}}^{*}$, there exists $\beta \in \mathbb{F}_{q^{n}}^{*}$ such that $f(\beta) / \beta=a \alpha$ for some $a \in \mathbb{F}_{q}^{*}$. In fact, we will solve the following $q$-ary version of Problem [.1:

Problem 1.2. Determine all linearized polynomials $f(x)=\sum_{i=0}^{n-1} a_{i} x^{q^{i}} \in \mathbb{F}_{q^{n}}[x]$ such that $f(x)$ is a permutation of $\mathbb{F}_{q^{n}}$ and $f(x) / x$ is a permutation of $\mathbb{F}_{q^{n}}^{*} / \mathbb{F}_{q}^{*}$.

Received by the editors July 29, 2002.

2000 Mathematics Subject Classification. Primary 11T06; Secondary 51E20.

Key words and phrases. Finite field, linearized polynomial, permutation polynomial.

This research was supported by NSA grant MDA 904-02-1-0080.

(C)2003 American Mathematical Society 
We briefly review linearized polynomials over finite fields in Section 2. In particular, we prove a proposition that slightly generalizes Dickson's criterion for a linearized polynomial to be nonsingular. The proof of our solution to Problem 1.2 is in Section 3. In Section 4, we solve another problem about linearized polynomials over $\mathbb{F}_{2^{n}}$ which is similar and related to Problem 1.1

\section{LINEARIZED POLYNOMIALS}

Let $\mathbb{F}_{q}$ and $\mathbb{F}_{q^{n}}$ be finite fields with $q$ and $q^{n}$ elements respectively. The $\mathbb{F}_{q^{-}}$-linear maps from $\mathbb{F}_{q^{n}}$ to $\mathbb{F}_{q^{n}}$ are precisely linearized polynomials

$$
f(x)=a_{0} x+a_{1} x^{q}+\cdots+a_{n-1} x^{q^{n-1}} \in \mathbb{F}_{q^{n}}[x] .
$$

Define

$$
A(f)=\left[\begin{array}{cccc}
a_{0} & a_{1} & \cdots & a_{n-1} \\
a_{n-1}^{q} & a_{0}^{q} & \cdots & a_{n-2}^{q} \\
\vdots & \vdots & \ddots & \vdots \\
a_{1}^{q^{n-1}} & a_{2}^{q^{n-1}} & \cdots & a_{0}^{q^{n-1}}
\end{array}\right] .
$$

It is well known that $f: \mathbb{F}_{q^{n}} \rightarrow \mathbb{F}_{q^{n}}$ is a permutation polynomial if and only if $\operatorname{det} A(f) \neq 0$ ([3], p. 66 or [4], p. 361). The following proposition slightly generalizes this criterion.

Proposition 2.1. In the above notation, we have

$$
\operatorname{rank} A(f)=\operatorname{dim}_{\mathbb{F}_{q}} f\left(\mathbb{F}_{q^{n}}\right) .
$$

Proof. Let

$$
V=\left\{\left[\begin{array}{c}
z \\
z^{q} \\
\vdots \\
z^{q^{n-1}}
\end{array}\right]: z \in \mathbb{F}_{q^{n}}\right\} \subset \mathbb{F}_{q^{n}}^{n}
$$

and define an $\mathbb{F}_{q}$-isomorphism

$$
\begin{array}{cl}
\iota: \mathbb{F}_{q^{n}} & \longrightarrow \\
z & \longmapsto\left[\begin{array}{c}
V \\
z \\
z^{q} \\
\vdots \\
z^{q^{n-1}}
\end{array}\right] .
\end{array}
$$

Note that the $\mathbb{F}_{q^{n}}$-linear map $A(f): \mathbb{F}_{q^{n}} \rightarrow \mathbb{F}_{q^{n}}$ satisfies $A(f)(V) \subset V$. Furthermore, we have the following commutative diagram:

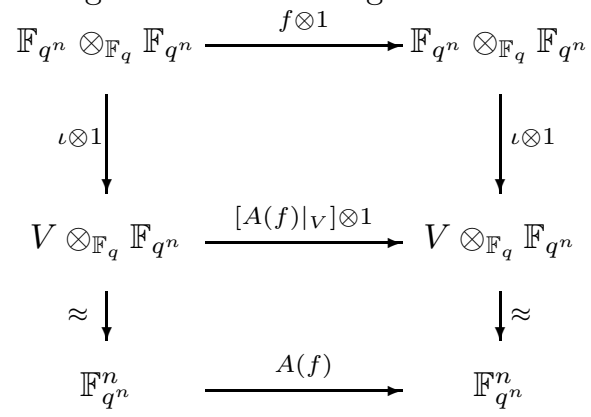


Therefore,

$$
\begin{aligned}
\operatorname{rank}(A(f)) & =\operatorname{dim}_{\mathbb{F}_{q^{n}}}\left(A(f)\left(\mathbb{F}_{q^{n}}^{n}\right)\right) \\
& =\operatorname{dim}_{\mathbb{F}_{q^{n}}}\left[(\iota \otimes 1) \circ(f \otimes 1)\left(\mathbb{F}_{q^{n}} \otimes_{\mathbb{F}_{q}} \mathbb{F}_{q^{n}}\right)\right] \\
& \left.=\operatorname{dim}_{\mathbb{F}_{q^{n}}}\left[f\left(\mathbb{F}_{q^{n}}\right) \otimes_{\mathbb{F}_{q}} \mathbb{F}_{q^{n}}\right)\right] \\
& =\operatorname{dim}_{\mathbb{F}_{q}} f\left(\mathbb{F}_{q^{n}}\right) .
\end{aligned}
$$

\section{Solution to Problem 1.2}

Let $q$ be a prime power and $n$ a positive integer.

Lemma 3.1. Let $f(x)=\sum_{i=0}^{n-1} a_{i} x^{q^{i}} \in \mathbb{F}_{q^{n}}[x]$ be a polynomial in Problem 1.2. Then the determinants of the principal submatrices of

$$
A(f)=\left[\begin{array}{cccc}
a_{0} & a_{1} & \cdots & a_{n-1} \\
a_{n-1}^{q} & a_{0}^{q} & \cdots & a_{n-2}^{q} \\
\vdots & \vdots & \ddots & \vdots \\
a_{1}^{q^{n-1}} & a_{2}^{q^{n-1}} & \cdots & a_{0}^{q^{n-1}}
\end{array}\right]
$$

of size $m \times m(1 \leq m \leq n-1)$ are all 0 .

Proof. Let

$$
D(x)=\left|\begin{array}{cccc}
a_{0}+x & a_{1} & \cdots & a_{n-1} \\
a_{n-1}^{q} & \left(a_{0}+x\right)^{q} & \cdots & a_{n-2}^{q} \\
\vdots & \vdots & \ddots & \vdots \\
a_{1}^{q^{n-1}} & a_{2}^{q^{n-1}} & \cdots & \left(a_{0}+x\right)^{q^{n-1}}
\end{array}\right| \in \mathbb{F}_{q^{n}}[x] .
$$

For each $b \in \mathbb{F}_{q^{n}}^{*}$, since $f(x) / x$ is a permutation of $\mathbb{F}_{q^{n}}^{*} / \mathbb{F}_{q}^{*}$, there exist $z \in \mathbb{F}_{q^{n}}^{*}$ and $\epsilon \in \mathbb{F}_{q}^{*}$ such that $\frac{f(z)}{z}=-\epsilon b$. Thus $z$ is a root of

$$
\left(a_{0}+\epsilon b\right) x+a_{1} x^{q}+\cdots+a_{n-1} x^{q^{n-1}} ;
$$

hence the polynomial in (3.1) is not a permutation polynomial of $\mathbb{F}_{q^{n}}$. It follows from Proposition 2.1 that $D(\epsilon b)=0$. Therefore, for every $b \in \mathbb{F}_{q^{n}}^{*}, \prod_{\epsilon \in \mathbb{F}_{q}^{*}} D(\epsilon b)=0$, which implies that

$$
\prod_{\epsilon \in \mathbb{F}_{q}^{*}} D(\epsilon x)=\delta\left(x^{q^{n}-1}-1\right)
$$

for some $\delta \in \mathbb{F}_{q^{n}}^{*}$. (In fact, $\delta=-1$, although this fact is not needed in the proof. This is because $D(0)$ is invariant under the Frobenius map of $\mathbb{F}_{q^{n}} / \mathbb{F}_{q}$ and $\left.-\delta=(D(0))^{q-1}=1\right)$.

Let $0 \leq i_{1}<i_{2}<\cdots<i_{m} \leq n-1$ with $1 \leq m \leq n-1$. Write $\{0, \cdots, n-1\} \backslash$ $\left\{i_{1}, \cdots, i_{m}\right\}=\left\{j_{1}, \cdots, j_{s}\right\}$ with $0 \leq j_{1}<\cdots<j_{s} \leq n-1$. Consider the coefficient of $x^{(q-1) q^{j_{1}}+\cdots+(q-1) q^{j_{s}}}$ in

$$
\prod_{\epsilon \in \mathbb{F}_{q}^{*}} D(\epsilon x)=\prod_{\epsilon \in \mathbb{F}_{q}^{*}}\left|\begin{array}{cccc}
a_{0}+\epsilon x & a_{1} & \cdots & a_{n-1} \\
a_{n-1}^{q} & a_{0}^{q}+\epsilon x^{q} & \cdots & a_{n-2}^{q} \\
\vdots & \vdots & \ddots & \vdots \\
a_{1}^{q^{n-1}} & a_{2}^{q^{n-1}} & \cdots & a_{0}^{q^{n-1}}+\epsilon x^{q^{n-1}}
\end{array}\right| .
$$


By the uniqueness of the $q$-adic expansion of $(q-1) q^{j_{1}}+\cdots+(q-1) q^{j_{s}}$, we see that this coefficient equals

$$
\left[\operatorname{det}\left(A(f)\left(i_{1}, \cdots, i_{m}\right)\right)\right]^{q-1} \prod_{\epsilon \in \mathbb{F}_{q}^{*}} \epsilon^{s}=\left[\operatorname{det}\left(A(f)\left(i_{1}, \cdots, i_{m}\right)\right)\right]^{q-1}(-1)^{s},
$$

where $A(f)\left(i_{1}, \cdots, i_{m}\right)$ is the principal submatrix of $A(f)$ with row and column indices $i_{1}, \cdots, i_{m}$, namely, the submatrix of $A(f)$ obtained by deleting rows and columns with indices other than $i_{1}, \cdots, i_{m}$. Comparing the coefficients of $x^{(q-1) q^{j_{1}}+\cdots+(q-1) q^{j_{s}}}$ in the two sides of (3.2), we have $\operatorname{det}\left(A(f)\left(i_{1}, \cdots, i_{m}\right)\right)$ $=0$.

Theorem 3.2. The polynomials in Problem 1.2 are exactly the ones of the form $f(x)=a x^{q^{k}}$ where $a \in \mathbb{F}_{q^{n}}^{*}$ and $k$ is a positive integer such that $(k, n)=1$.

Proof. Let $f(x)=a_{0} x+a_{1} x^{q}+\cdots+a_{n-1} x^{q^{n-1}} \in \mathbb{F}_{q^{n}}[x]$ be a polynomial in Problem 1.2 It suffices to show that $f(x)$ has exactly one nonzero coefficient. By Lemma 3.1, the determinants of principal submatrices of

$$
A(f)=\left[\begin{array}{cccc}
a_{0} & a_{1} & \cdots & a_{n-1} \\
a_{n-1}^{q} & a_{0}^{q} & \cdots & a_{n-2}^{q} \\
\vdots & \vdots & \ddots & \vdots \\
a_{1}^{q^{n-1}} & a_{2}^{q^{n-1}} & \cdots & a_{0}^{q^{n-1}}
\end{array}\right]
$$

of sizes $1 \times 1,2 \times 2, \cdots,(n-1) \times(n-1)$ are all 0 . Observe that

$$
A(f)=\left[b_{i j}\right]_{0 \leq i, j \leq n-1}
$$

where

$$
b_{i j}=0 \text { if and only if } a_{j-i}=0,
$$

where the subscript is taken modulo $n$.

We claim that if $i_{1}+\cdots+i_{m} \equiv 0(\bmod n)(1 \leq m \leq n-1)$, then

$$
a_{i_{1}} \cdots a_{i_{m}}=0 .
$$

To prove (3.5), we use induction on $m$. The case $m=1$ is obvious. Assume to the contrary that $i_{1}+\cdots+i_{m} \equiv 0(\bmod n)$ but $a_{i_{1}} \cdots a_{i_{m}} \neq 0$. We may assume that $0, i_{1}, i_{1}+i_{2}, \cdots, i_{1}+\cdots+i_{m-1}$ are all distinct modulo $n$. (Otherwise, $i_{s}+\cdots+i_{t} \equiv 0$ $(\bmod n)$ for some $1 \leq s<t \leq m-1$. By the induction hypothesis, $a_{i_{s}} \cdots a_{i_{t}}=0$, which is a contradiction.) Consider the principal submatrix of $A(f)$ with row and column indices $j_{0}=0, j_{1}=i_{1}, j_{2}=i_{1}+i_{2}, \cdots, j_{m-1}=i_{1}+\cdots+i_{m-1}$ :

$$
B=\left[\begin{array}{ccccc}
0 & b_{0 j_{1}} & b_{0 j_{2}} & \cdots & b_{0 j_{m-1}} \\
b_{j_{1} 0} & 0 & b_{j_{1} j_{2}} & \cdots & b_{j_{1} j_{m-1}} \\
b_{j_{2} 0} & b_{j_{2} j_{1}} & 0 & \cdots & b_{j_{2} j_{m-1}} \\
\vdots & \vdots & \vdots & \ddots & \vdots \\
b_{j_{m-1} 0} & b_{j_{m-1} j_{1}} & b_{j_{m-1} j_{2}} & \cdots & 0
\end{array}\right] .
$$

Since $a_{i_{1}}, \cdots, a_{i_{m}}$ are all nonzero, by (3.4), $b_{0 j_{1}}, b_{j_{1} j_{2}}, \cdots, b_{j_{m-2} j_{m-1}}, b_{j_{m-1} 0}$ are all nonzero. Since all $2 \times 2$ principal submatrices of $B$ have determinant $0, b_{j_{1} 0}=$ 
$b_{j_{2} j_{1}}=\cdots=b_{j_{m-1} j_{m-2}}=0$. Since all $3 \times 3$ principal submatrices of $B$ have determinant 0 , it follows that $b_{j_{2} 0}=b_{j_{3} j_{1}}=\cdots=b_{j_{m-1} j_{m-3}}=0$. (For example,

$$
0=\left|\begin{array}{ccc}
0 & b_{0 j_{1}} & b_{0 j_{2}} \\
0 & 0 & b_{j_{1} j_{2}} \\
b_{j_{2} 0} & 0 & 0
\end{array}\right|=b_{0 j_{1}} b_{j_{1} j_{2}} b_{j_{2} 0}
$$

implies that $b_{j_{2} 0}=0$.) In the same way, by considering principal submatrices of $B$ up to size $(m-1) \times(m-1)$, we conclude that

$$
B=\left[\begin{array}{cccccc}
0 & b_{0 j_{1}} & * & \cdots & * & * \\
0 & 0 & b_{j_{1} j_{2}} & \cdots & * & * \\
0 & 0 & 0 & \cdots & * & * \\
\vdots & \vdots & \vdots & \ddots & \vdots & \vdots \\
0 & 0 & 0 & \cdots & 0 & b_{j_{m-2} j_{m-1}} \\
b_{j_{m-1} 0} & 0 & 0 & \cdots & 0 & 0
\end{array}\right] .
$$

It follows that $b_{0 j_{1}} b_{j_{1} j_{2}} \cdots b_{j_{m-2} j_{m-1}} b_{j_{m-1} 0}=\operatorname{det} B=0$, which is a contradiction. Thus (3.5) is proved.

Assume that $a_{k} \neq 0$ for some $1 \leq k \leq n-1$. We claim that $(k, n)=1$. Otherwise, there is an integer $1 \leq l \leq n-1$ such that $l k \equiv 0(\bmod n)$. By (3.5), we have

$$
\underbrace{a_{k} \cdots a_{k}}_{l}=0
$$

which is a contradiction. For any $1 \leq i \leq n-1$ with $i \neq k$, we can write $i \equiv-j k$ $(\bmod n)$ with $1 \leq j \leq n-2$. By (3.5) again, we have

$$
a_{i} \underbrace{a_{k} \cdots a_{k}}_{j}=0
$$

which implies that $a_{i}=0$. Thus $a_{k}$ is the only nonzero coefficient of $f$ and the proof of the theorem is complete.

\section{A Related PRoblem}

We consider another problem similar to Problem 1.1

Problem 4.1. Determine all linearized polynomials $f(x)=\sum_{i=0}^{n-1} a_{i} x^{2^{i}} \in \mathbb{F}_{2^{n}}[x]$ such that for any $c \in \mathbb{F}_{2^{n}}$, the range of $f(x)+c x$ has dimension $\geq n-1$ over $\mathbb{F}_{2}$.

We mention that Problem 4.1 is related to a construction of partial difference sets in $\mathbb{Z}_{4}^{n} \times \mathbb{Z}_{2}^{n}$ ([1]). The solution of Problem 4.1] is similar to that of Problem 1.1

Theorem 4.2. The polynomials in Problem 4.1 are exactly the ones of the form $f(x)=a x^{2^{k}}+b x$ where $a \in \mathbb{F}_{2^{n}}^{*}, b \in \mathbb{F}_{2^{n}}$ and $(k, n)=1$.

Proof. First assume that $f(x)=a x^{2^{k}}+b x$ with $a \in \mathbb{F}_{2^{n}}^{*}, b \in \mathbb{F}_{2^{n}}$ and $(k, n)=1$. Then for any $c \in \mathbb{F}_{2^{n}}, f(x)+c x=a x\left(x^{2^{k}-1}+\frac{b+c}{a}\right)$ has at most two roots in $\mathbb{F}_{2^{n}}$. Thus the range of $f(x)+c x$ has dimension $\geq n-1$ over $\mathbb{F}_{2}$.

Now assume that $f(x)=\sum_{i=0}^{n-1} a_{i} x^{2^{i}} \in \mathbb{F}_{2^{n}}[x]$ is a polynomial in Problem 4.1 For each $c \in \mathbb{F}_{2^{n}}, f(x)+c x$ has at most one zero in $\mathbb{F}_{2^{n}}^{*}$, i.e., $\frac{f(x)}{x}=c$ has at most one solution in $\mathbb{F}_{2^{n}}^{*}$. Thus the map

$$
\begin{aligned}
\psi: \mathbb{F}_{2^{n}}^{*} & \longrightarrow \mathbb{F}_{2^{n}} \\
x & \longmapsto \frac{f(x)}{x}
\end{aligned}
$$


is one-to-one. Let $\mathbb{F}_{2^{n}} \backslash \psi\left(\mathbb{F}_{2^{n}}^{*}\right)=\{b\}$. Then $f(x)+b x$ has no root in $\mathbb{F}_{2^{n}}^{*}$, hence is a permutation polynomial of $\mathbb{F}_{2^{n}}$. Furthermore, $\frac{f(x)+b x}{x}=\frac{f(x)}{x}+b$ is a permutation of $\mathbb{F}_{2^{n}}^{*}$. By Theorem 3.2, $f(x)+b x=a x^{2^{k}}$ where $a \in \mathbb{F}_{2^{n}}^{*}$ and $(k, n)=1$.

Finally, we remark that we have not found a $q$-ary version of Theorem 4.2 .

\section{REFERENCES}

[1] J. Davis and Q. Xiang, A family of partial difference sets with Denniston parameters in nonelementary abelian 2-groups, European J. Combin. 21 (2000), 981 - 988. MR 2002a:05043

[2] P. Dembowski, Finite Geometries, Ergebnisse der Mathematik und ihrer Grenzgebiete, Band 44, Springer-Verlag, New York, 1968. MR 38:1597

[3] L. E. Dickson, Linear Groups: With an Exposition of the Galois Field Theory, Dover, New York, 1958. MR 21:3488

[4] R. Lidl and H. Niederreiter, Finite Fields, Addison-Wesley, Reading, MA, 1983. MR 86c:11106

[5] S. E. Payne, Affine representations of generalized quadrangles, J. Algebra 16 (1970), 473 485. MR 42:8381

[6] S. E. Payne, Linear transformations of a finite field, Amer. Math. Monthly 78 (1971), 659 660.

Department of Mathematics and Statistics, Wright State University, Dayton, Ohio 45435

E-mail address: xhou@euler.math.wright.edu

Current address: Department of Mathematics, University of South Florida, Tampa, Florida 33620 